\title{
Thin broadband noise absorption through acoustic reactance control by electro-mechanical coupling without sensor
}

\author{
Yumin Zhang, ${ }^{\text {a) }}$ Yum-Ji Chan, ${ }^{\text {b) }}$ and Lixi Huang \\ Aerodynamics and Acoustics Laboratory, HKU Zhejiang Institute of Research and Innovation, \\ Department of Mechanical Engineering, The University of Hong Kong, Hong Kong SAR, China
}

(Received 11 July 2013; revised 24 March 2014; accepted 2 April 2014)

\begin{abstract}
Broadband noise with profound low-frequency profile is prevalent and difficult to be controlled mechanically. This study demonstrates effective broadband sound absorption by reducing the mechanical reactance of a loudspeaker using a shunt circuit through electro-mechanical coupling, which induces reactance with different signs from that of loudspeaker. An RLC shunt circuit is connected to the moving coil to provide an electrically induced mechanical impedance which counters the cavity stiffness at low frequencies and reduces the system inertia above the resonance frequency. A sound absorption coefficient well above 0.5 is demonstrated across frequencies between 150 and $1200 \mathrm{~Hz}$. The performance of the proposed device is superior to existing passive absorbers of the same depth $(60 \mathrm{~mm})$, which has lower frequency limits of around $300 \mathrm{~Hz}$. A passive noise absorber is further proposed by paralleling a micro-perforated panel with shunted loudspeaker which shows potentials in absorbing band-limit impulse noise.
\end{abstract}

(C) 2014 Acoustical Society of America. [http://dx.doi.org/10.1121/1.4871189]

PACS number(s): 43.50.Gf, 43.55.Ev, 43.38.Ja [KVH]

Pages: $2738-2745$

\section{INTRODUCTION}

Broadband noise is prevalent in our living environment such as impulse noise from construction sites. Its lowfrequency content is difficult to be controlled by passive means in compact devices. Passive noise control is still the preferred approach because active noise control (ANC) (Nelson and Elliott, 1992; Hansen, 2002) suffers from issues such as cost, performance, and reliability. Passive noise control can be achieved through sound reflection and sound absorption: While the sound-reflection strategy is effective in particular applications such as duct noise control, the sound-absorption counterpart (Delany and Bazley, 1970; Ingard, 2008; Mechel, 2001) is more versatile in practice. Sound absorption is most effective around the resonance frequency of the sound absorber, where reactance vanishes. At extremely low frequencies, the reactance is dominated by the stiffness of air cavities and sound absorption performance is poor, and at high frequencies, mass effect dominates and sound absorption becomes ineffective. This work aims to extend the lower limit of effective sound absorption by suppressing the system reactance through electromechanical coupling. Such coupling occurs as electric current is developed in the moving coil-which is the voice coil in a loudspeaker-when a moving coil cuts through a magnetic field. At the same time, a mechanical reaction force, known as the electro-magnetic force, is generated. The electro-magnetic force can alter the mechanical impedance (thus sound-absorption characteristics) of a spring-mass dynamic system. In this study, a moving-coil loudspeaker

\footnotetext{
${ }^{\text {a) }}$ Author to whom correspondence should be addressed. Electronic mail: yumin@connect.hku.hk

${ }^{b}$ Current address: Department of Mechanical Engineering, National Chung Hsing University, Taichung, Taiwan.
}

driver unit is chosen as it offers an assembly of a springmass system and an electro-mechanical coupler. Unlike typical loudspeakers, the loudspeaker in the current study functions as a "passive" device, without an electrical input signal. To avoid confusion between the proposed soundabsorption device and typical loudspeakers, the proposed sound absorber is denoted shunted electro-magnetic diaphragm (SEMD) throughout the text. A hybrid absorber through installing SEMD with a micro-perforated panel (MPP) in parallel is further proposed to advance highfrequency absorption. The hybrid absorber is found to be capable of reducing the magnitude of acoustics impulse in calculations and it hints that such a device can protect the human ear exposed to impact noise (Coles et al., 1968).

Electrical and mechanical impedances are coupled in electro-mechanical devices (Hunt, 1982) such as condenser microphones and piezoelectric patches used to absorb vibration energy of plates. It is known that shunt circuit is a convenient method to alter the mechanical impedance in coupled systems. Forward (1979) and Swigert and Forward (1981) presented an electronic circuit producing mechanical damping in vibration control first. Hagood and von Flotow (1991) introduced shunt circuits to piezoelectric patches to improve vibration absorption performance. Behrens et al. (2003) used capacitive shunting to suppress multiple resonant modes of a piezoelectric-laminated plate. Fukada et al. (2005) went as far as creating negative elasticity for curved polyvinylidene fluoride (PVDF) and piezoelectric ceramic (PZT) patches. Kodama et al. (2008) and Tajitsu et al. (2007) also applied shunt circuit to control vibration but their works were mainly motivated by the insulation of sound radiated from vibrating structures. Tang and Wang (2001) pointed out that negative capacitor shunting would benefit both passive damping and active control authority in a hybrid network. Kim and Lee (2002) used a resonant shunt circuit to create 
damping of the host panel for the similar purpose of suppressing its noise radiation. Kim and Jung (2006) improved the design by using an identical patch of piezoelectric material as the canceling impedance to account for the complex frequency dependency of the material. Chang et al. (2010) shunted MPP coated with piezoelectric materials to absorb low-frequency noise.

The use of shunt circuits in controlling airborne noise has been relatively rare due to the apparent density mismatch between air and the vibrating structure to which a shunt circuit is attached. Recent literature reports studies of using shunted loudspeaker to control narrow band noise. Fleming et al. (2007) used a moving-coil loudspeaker as a side-branch Helmholtz resonator to suppress the duct acoustics modes. Pietrzko and Mao (2009) also attempted to use a shunted loudspeaker to absorb sound with a configuration similar the one discussed in the present study. Cheung (2010) studied shunted loudspeaker as Helmholtz resonator to reflect low-frequency duct noise. Lissek and Meynial (2003) and Lissek et al. (2009), who were among the first to develop such shunt circuit techniques, used a shunted loudspeaker to control wall acoustic impedance. Their further work (Lissek et al., 2011; Boulandet and Lissek, 2010) reported good low-frequency absorption in a relative narrow frequency-band, which is achieved by resonance of shunted loudspeaker. Zhang (2012) studied the effects of different electrical components in the shunt circuit of a loudspeaker and proposed an innovative strategy to suppress the system dynamic mass for both improved low-frequency and high-frequency performance.

Although past research provided great insight on using shunted loudspeaker as noise absorber, it is stressed here that the experimental results of mentioned work (Fleming et al., 2007; Pietrzko and Mao, 2009; Lissek et al., 2009; Boulandet and Lissek, 2010) did not produce stand-alone units. Their absorbers were constructed according to the absorption mechanism of resonators and their broadband results were determined by sensors and active controllers. These characteristics bear resemblance to an ANC system. ANC can be considered as a method to modify the mechanical impedance of the "sound absorber," which is commonly known as the secondary source.

Although the objectives are similar [for example, a piston mounted on the side-wall of ducts should present zero resistance, zero stiffness, and negative effective mass to achieve total sound reflection (Huang, 2000; Lissek et al. 2011)], the loudspeaker in ANC and an SEMD are actuated by different mechanisms. In ANC systems, the secondary loudspeaker is controlled by sensors and filters (Cobo et al., 2003; Cobo et al., 2004), while no sensors are required in SEMDs. The SEMD achieves broadband noise absorption by reactance suppression. Current results are compared to those reported in the literature in Sec. IV.

While the principle of modifying mechanical impedance in electro-mechanically coupled structures using electrical methods is known, this work investigates whether a shunted loudspeaker can achieve broadband absorption through reducing acoustic reactance, in particular, whether it can extend the lower frequency limit of effective broadband sound absorption. In what follows, the design of the shunt circuit to reduce reactance of loudspeaker is described in
Sec. II, and the experimental results are shown in Sec. III, where the key finding that the lower frequency limit of sound absorption is extended by an octave band when compared with traditional absorbers using porous material or microperforated panels is shown. The discussion in Sec. IV, explores the possibility of further bandwidth extension by using parallel absorbers and the proposed absorber is shown to be capable to control impulse noise. Conclusions are drawn in Sec. V.

\section{THEORETICAL MODEL AND SHUNT CIRCUIT DESIGN}

\section{A. Dynamics model of the shunted loudspeaker}

The layout of the proposed SEMD is shown in Fig. 1. A moving-coil loudspeaker is installed in a sealed rectangular box, and the assembly forms the sample-end of a standard impedance tube. The terminals of the loudspeakers are connected to a shunt circuit. With the motion of the diaphragm dictated by the shunt circuit, it is used as a sound absorber and called an SEMD.

The loudspeaker without the shunt circuit is analyzed first. The moving parts of the loudspeaker, which includes the diaphragm and the moving-coil, is considered a single degree-of-freedom, spring-mass system below the frequency of high-order mode cut-on for the diaphragm under linear hypothesis (Eargle, 2003). The mechanical impedance of an open-terminal loudspeaker in a sealed cavity with depth $D$ is given as follows:

$$
Z_{0}=\delta+i\left[m \omega-\kappa / \omega-Z_{\mathrm{air}} \cot \left(\omega D / c_{0}\right)\right]
$$

where $\omega$ is the angular frequency, $c_{0}$ is the speed of sound in air, $m, \delta, \kappa$ are the effective mass, damping, and stiffness of the moving-coil loudspeaker, respectively, $Z_{\mathrm{air}}=\rho_{0} c_{0} A$ is the acoustic impedance of air, and $A$ is the cross section area of the impedance tube. The terms in the square brackets in Eq. (1) are collectively called the acoustic reactance. When such a loudspeaker is installed at the end of an impedance tube, the normal-incidence sound absorption coefficient is

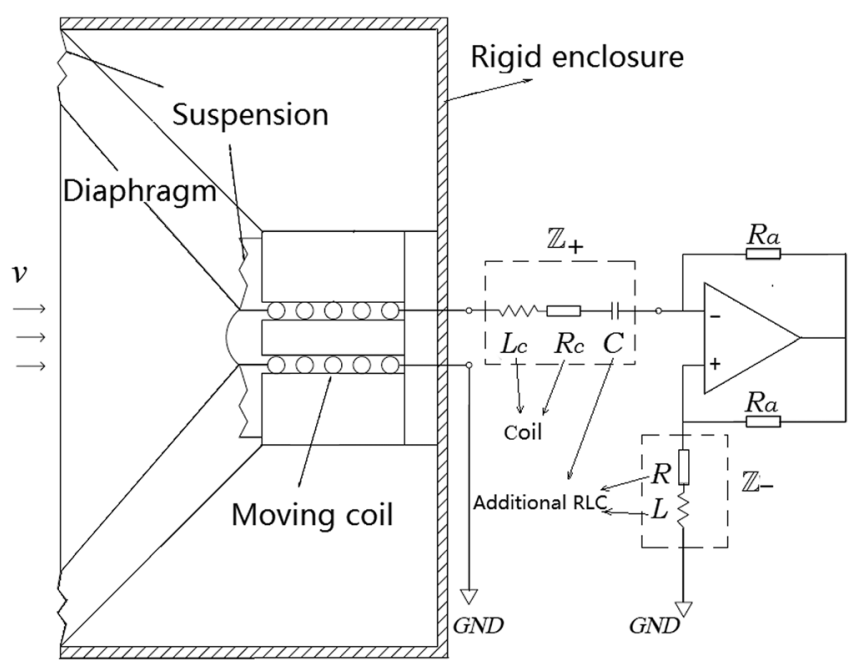

FIG. 1. Moving-coil loudspeaker with shunt circuit with intrinsic coil resistance is $R_{c}$ and inductance $L_{c}$. 


$$
\alpha(\omega)=1-\left|\frac{Z-Z_{\text {air }}}{Z+Z_{\text {air }}}\right|^{2}=\frac{4 Z_{\text {air }} \operatorname{Re}(Z)}{\left[Z_{\text {air }}+\operatorname{Re}(Z)\right]^{2}+[\operatorname{Im}(Z)]^{2}},
$$

where $Z=Z_{0}$ is the acoustic impedance over the loudspeaker diaphragm. Equations (1) and (2) show that perfect sound absorption requires zero reactance, $\operatorname{Im}(Z)=0$, and that the resistance equals that of air, $\operatorname{Re}(Z)=Z_{\text {air }}$. The reactance condition of $\operatorname{Im}(Z)=0$ is impossible to achieve when frequency approaches zero, $\omega \rightarrow 0$. As shown in Eq. (1), $\lim _{\omega \rightarrow 0} \operatorname{Im}\left(Z_{0}\right) \rightarrow \infty$ due to both the suspension stiffness term $\kappa / \omega$ and the cavity stiffness term $\cot \left(\omega D / c_{0}\right)$.

When the terminals of a loudspeaker are connected to a shunt circuit, a SEMD is created. The electrical load, denoted by $\mathbb{Z}$, brings additional mechanical impedance of $\Delta Z=(B l)^{2} / \mathbb{Z}$ (Hunt, 1982) where $B l$ is the force factor of the moving-coil loudspeaker and $\mathbb{Z}=\mathbb{Z}_{+}-\mathbb{Z}_{-}$is the total electrical impedance. The mechanical impedance of the SEMD becomes

$$
Z(\omega)=Z_{0}(\omega)+\Delta Z(\omega)=Z_{0}(\omega)+(B l)^{2} / \mathbb{Z} .
$$

In addition, the layout in Fig. 1 also includes a negative impedance converter (NIC) (Chen, 2003) is employed in the current test rig; with $\mathbb{Z}_{+}$being the inverting input to the operational amplifier, which is always positive, and $\mathbf{Z}_{-}$as the non-inverting input with ground. The use of $\mathbf{Z}_{-}$with the NIC circuit can create net electrical impedance, denoted $\mathbf{Z}$, beyond the range offered in commercially available movingcoil loudspeakers.

Coil resistance and inductance are denoted by $R_{C}$ and $L_{c}$, respectively. In the circuit shown in Fig. 1, a capacitor $C$ is attached to the coil. The net impedance, denoted $\mathbf{Z}$, is the impedance subtracted by the total impedance added to the NIC resistance, which is

$$
\mathbb{Z}=\left[R_{c}+i \omega L_{c}+(i \omega C)^{-1}\right]-(R+i \omega L)=\mathbb{Z}_{+}-\mathbb{Z}_{-} .
$$

The same argument can be made on each component of impedance to obtain $\Delta R=R_{c}-R, \Delta L=L_{c}-L$. When an acoustic wave reaches the diaphragm, the diagram will acquire a velocity $v$, and a voltage of $B l v$ is induced over the length of the coil. As the induced electrical current is $I=B l v / \mathbb{Z}$, the electro-magnetic force is $F=B l I=v(B l)^{2} / \mathbb{Z}$. The equivalent mechanical impedance induced by the circuit, $F / v$, is thus $(B l)^{2} / \mathbb{Z}$. By tuning $\mathbb{Z}$, a desirable acoustic impedance may be achieved. It should be noted that the NIC circuit is powered by DC sources and is therefore an active circuit, and stability issues need to be considered. For example, when the total electrical resistance becomes negative, $\Delta R=R_{c}-R<0$, while the capacitance and inductance remain positive, the circuit will be unstable. It is found in the current study (Sec. II B) that good absorbers can be obtained with all three parameters remaining positive $(\Delta R>0, \Delta L>0, C>0)$ and a stable system is guaranteed (Zhang, 2012). In fact, such a system can be constructed completely passive without NIC, as the NIC is used mainly for the ease of fine-tuning in this study. This point will be discussed again at the end of Sec. III.

\section{B. Impedance design}

Substitution of $Z$ in Eq. (2) by that in Eq. (3) yields

$$
\alpha(\omega)=\frac{4 Z_{\mathrm{air}}\left[(B l)^{2} \operatorname{Re}\left(Z^{-1}\right)+\delta\right]}{\left[(B l)^{2} \operatorname{Re}\left(Z^{-1}\right)+\delta+Z_{\mathrm{air}}\right]^{2}+\left[(B l)^{2} \operatorname{Im}\left(Z^{-1}\right)+m \omega-\kappa / \omega-\cot \left(\omega D / c_{0}\right)\right]^{2}} .
$$

To improve the low-frequency absorption, the electrical impedance $\mathbf{Z}$ can be designed to suppress the system reactance at low frequencies. $\mathbf{Z}$ may also improve the matching of system mechanical resistance to that of air. The electrical impedance $\mathbf{Z}$ and admittance $\mathbf{Z}^{-1}$ for the circuit in Fig. 1, are written as follows

$$
\begin{aligned}
& Z=\Delta R-i \omega q, \quad q=\left(C \omega^{2}\right)^{-1}-\Delta L, \\
& Z^{-1}=\frac{\Delta R+i \omega q}{\Delta R^{2}+\omega^{2} q^{2}} .
\end{aligned}
$$

The electrically induced extra acoustical impedance $\Delta Z$ is divided into real (resistance) and imaginary (reactance) parts,

$$
\begin{gathered}
\Delta Z_{r} \equiv \operatorname{Re}(\Delta Z)=\frac{(B l)^{2} \Delta R}{\Delta R^{2}+\omega^{2} q^{2}}, \\
\Delta Z_{i} \equiv \operatorname{Im}(\Delta Z)=\frac{(B l)^{2} \omega q}{\Delta R^{2}+\omega^{2} q^{2}} .
\end{gathered}
$$

According to Eq. (1), the reactance of the loudspeaker is negative, $\operatorname{Im}\left(Z_{0}\right)<0$, when $\omega<\omega_{r}$, where $\omega_{r}$ is the natural frequency of the open-terminal loudspeaker with the back cavity, and positive when $\omega>\omega_{r}$. To decrease the magnitude of the loudspeaker reactance, the electrically induced reactance $\Delta Z_{i}$ should counter the influence of $\operatorname{Im}\left(Z_{0}\right)$,

$$
\Delta Z_{i} \begin{cases}>0, & \omega<\omega_{r} \\ <0, & \omega>\omega_{r} .\end{cases}
$$

Equation (7) shows that $q$ controls the sign of the induced reactance $\Delta Z_{i}$, which can be tuned to any desirable value by the capacitor and the NIC circuit. To satisfy Eq. (8), $q$ has to be positive at low frequencies and negative at high frequencies. $q$ changes sign around the circuit resonant frequency, denoted as $\omega_{c}$ and defined in Eq. (9),

$$
\omega_{c}=1 / \sqrt{C \Delta L} .
$$


Figure 2 gives a typical set of spectra of $\Delta Z(\omega)$ (dashed lines) and they are compared with a mechanical impedance $Z_{\text {mech }}$ (solid lines) with $m=2 \mathrm{~g}, \delta=3 \mathrm{Ns} / \mathrm{m}$, and $\kappa=10000 \mathrm{~N} / \mathrm{m}$. On one hand, the shape of the induced reactance $\Delta Z_{i}$ shown in Fig. 2(b) is explained by inspecting the second formula in Eq. (7) together with the definition of $q$ in Eq. (6). $\Delta Z_{i}=0$ at three locations:

(1) at $\omega=0$, the circuit is effectively disconnected by the capacitor, hence $q=\infty, \Delta Z_{i}=0$;

(2) at electrical resonance $\left(\omega=\omega_{c}\right), q=0$ thus $\Delta Z_{i}=0$;

(3) at $\omega \rightarrow \infty$, the serial inductance $\Delta L$ effectively disconnects the shunt circuit, giving zero electrical admittance, hence $\Delta Z_{i}=0$ again.

$\Delta Z_{i}$ is positive and has a maximum within $\omega \in\left(0, \omega_{c}\right)$ when $\omega>\omega_{c}, q<0$, and $\Delta Z_{i}<0$. Therefore, there is a minimum of $\Delta Z_{i}$ within $\omega \in\left(\omega_{c}, \infty\right)$.

On the other hand, the induced damping, $\Delta Z_{r}$ shown in Fig. 2(a), is always positive. It vanishes when $\omega=0,+\infty$, and reaches its peak at the electrical resonance, $\omega=\omega_{c}$. These features are summarized in Table I. It is noted that the force factor of the loudspeaker, denoted $B l$, is a key loudspeaker parameter in impedance design. A high value of $B l$ may bring too much damping to the system and reactance control is difficult with a low value of $B l$.

At extremely low frequency, the circuit is effectively disconnected by the capacitor, hence $q=\infty, \Delta Z_{i}=0$. At the electrical resonance, $\omega=\omega_{c}$, the factor $q=0$ which gives $\Delta Z_{i}=0$. At $\Delta Z_{i}$ there is a peak value within $\omega \in$ $\left(0, \omega_{c}\right)$ when $\omega>\omega_{c}, q<0$, and $\Delta Z_{i}<0$. At very high frequencies, $\omega \rightarrow \infty$, the series inductance $\Delta L$ effectively disconnects the shunt circuit giving zero electrical admittance, hence $\Delta Z_{i}=0$. Therefore, there is a trough of $\Delta Z_{i}$ for $\omega \in\left(\omega_{c}, \infty\right)$. Overall, the curve of $\Delta Z_{i}(\omega)$ resembles that of a stretched sine curve from angle 0 to $2 \pi$. On the other hand, the induced damping, $\Delta Z_{r}$ shown in Fig. 2(a), is always positive. It vanishes when $\omega=0,+\infty$, and reaches its peak at
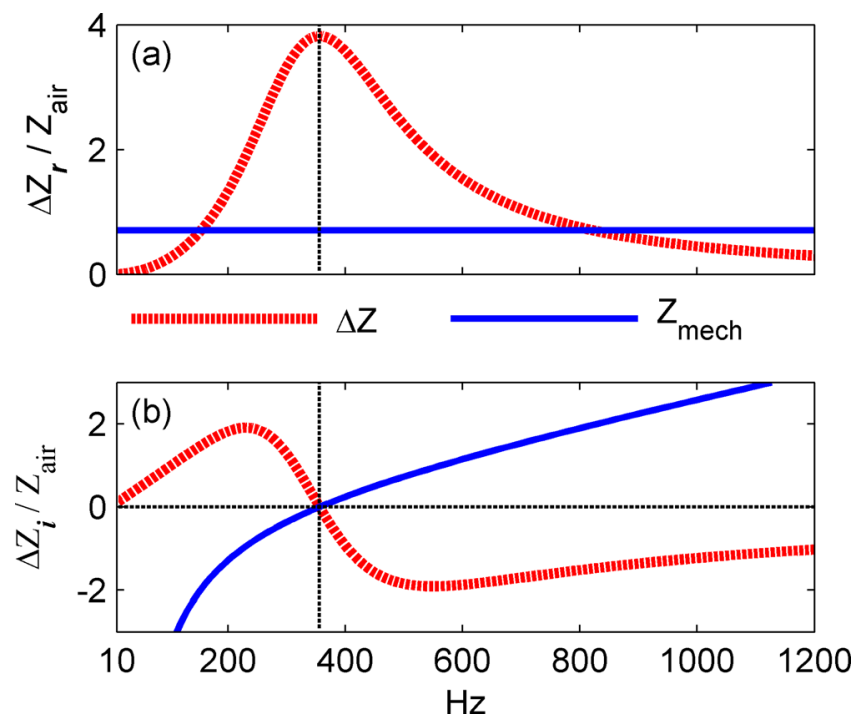

FIG. 2. (Color online) (a) Real and (b) imaginary parts of electrically induced impedance $\Delta Z$ (dashed lines) and mechanical impedance $Z_{\text {mech }}$ (solid lines). Parameters to obtain $\Delta Z: \Delta R=2 \Omega, \Delta L=1000 \mu \mathrm{H}, \quad \Delta L=200 \mu \mathrm{F}$, $f_{c}=355.9 \mathrm{~Hz}$; those for $Z_{\text {mech }}: m=2 \mathrm{~g}, \delta=3 \mathrm{Ns} / \mathrm{m}$, and $\kappa=10000 \mathrm{~N} / \mathrm{m}$.
TABLE I. Properties of electrically induced impedance as a function of frequency, $\Delta Z(\omega)$.

\begin{tabular}{llllll}
\hline \hline Frequency & $\omega=0$ & $0<\omega<\omega_{c}$ & $\omega=\omega_{c}$ & $\omega>\omega_{c}$ & $\omega \rightarrow \infty$ \\
\hline$\Delta Z_{r}$ & 0 & + & $\max$ & + & $\rightarrow 0$ \\
$\Delta Z_{+}$ & 0 & + & 0 & - & $\rightarrow 0$ \\
\hline \hline
\end{tabular}

the electrical resonance, $\omega=\omega_{c}$. These features are summarized in Table I.

\section{EXPERIMENTAL RESULTS}

The experimental setup is shown in Fig. 3. The D/A module (NI 9263) of a National Instrument CompactRIO ${ }^{\circledR}$ system generates a harmonic signal and it is amplified before being fed into the sound source, while the A/D module (NI 9232) converts the signal from microphones. The core of the test rig is an impedance tube of square cross section with sides of $100 \mathrm{~mm}$. The back side of the loudspeaker is sealed in a box, and the box is fixed at the right-hand end of the impedance tube.

To find the equivalent length of the air volume behind the diaphragm, the dimensions of the box are noted to be $170 \times 170 \times 30 \mathrm{~mm}$, thus $V_{\text {box }}=170 \times 170 \times 30=8.67$ $\times 10^{5} \mathrm{~mm}^{3}$. However, part of the $V_{\text {box }}$ is located in front of the diaphragm. Based on the measured dimensions of the loudspeaker cone, the volume, which consists of a "cylinder" and a "truncated cone," is

$$
\begin{aligned}
V_{\text {cone }}= & \left(\pi \times 82.5^{2}\right) \times 5.5+\frac{1}{3} \pi\left(60^{2}+10 \times 60+10^{2}\right) \\
& \times 24.5=2.28 \times 10^{5} \mathrm{~mm}^{3}
\end{aligned}
$$

Also, the volume of the Blu-tack for sealing purposes is estimated as $0.45 \times 10^{5} \mathrm{~m}^{3}$. As a result, the equivalent tube length behind the diaphragm is

$$
D=\frac{V_{\text {box }}-V_{\text {cone }}-0.45 \times 10^{5}}{100 \times 100} \approx 60 \mathrm{~mm} .
$$

The sample loudspeaker used is Visaton BG-17 whose technical data is shown in Table II. Note that the loudspeaker damping coefficient $\delta$ is a function of frequency. Also, as the coefficient may vary across products of the same model, the parameter is measured and is used throughout the current study. $\delta / \rho_{0} c_{0} A$ is shown in Fig. 4(b) and Fig. 5(b) and the value is 0.32 at $120 \mathrm{~Hz}$. The acoustic impedance of the opencircuit loudspeaker, which means the shunt circuit is disconnected, is measured using the standard two-microphone method (Chung and Blaser, 1980a,b) with harmonic excitation signals and is shown in Fig. 4.

Figure $4(a)$ is the sound absorption coefficient and Fig. 4(b) shows the real (solid line) and imaginary (dashed line) parts of the impedance of the open-circuit loudspeaker installed with a cavity of equivalent depth of $60 \mathrm{~mm}$. Below about $150 \mathrm{~Hz}$, the absorption performance is poor because of excessive acoustic reactance. Figure 4(b) shows that the reactance vanishes, $\operatorname{Im}\left(Z_{0}\right)=0$, around $375 \mathrm{~Hz}$, where an absorption peak is seen in Fig. 4(a). The second absorption 


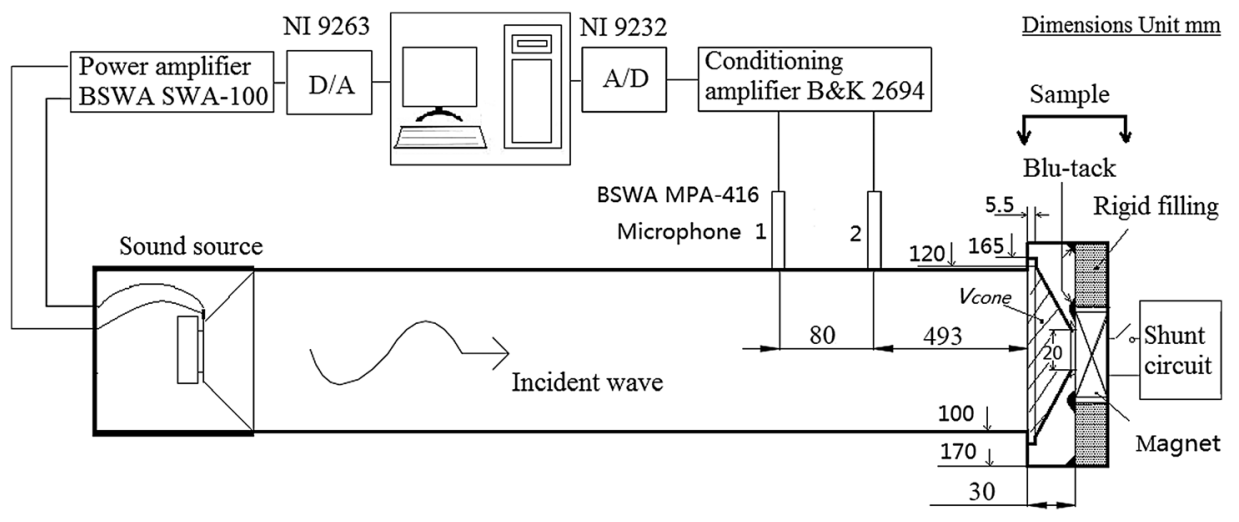

FIG. 3. Measurement setup.

peak is found around $650 \mathrm{~Hz}$ where the dimensionless acoustic resistance closely matches that of air, $\operatorname{Re}\left(Z_{0}\right) \approx 1$, as shown by the solid curve in Fig. 4(b). The absorption performance outside this narrow frequency range is poor mainly due to the large magnitude of acoustic reactance.

The purpose of the shunt circuit is to suppress the mechanical reactance and to adjust the mechanical resistance to a value closer to that of air. Although the circuit can be design to have a resonant frequency matching the mechanical counterpart, that is $\omega_{c}=\omega_{r}$, the frequency dependency of open-circuit reactance [Fig. 4(b), dashed line], and the electrically-induced reactance [Fig. 2(b)] are different. As a result, the circuit parameters are chosen to match the peak reactance positions in order to achieve reactance cancelation over a wide frequency band. This requires the circuit resonant frequency to be slightly below that of the mechanical counterpart, that is $\omega_{c}<\omega_{r}$. To test the effectiveness of this approach, three sets of circuit parameters are selected as shown in Table III and operational amplifier used is LM358 with $R_{a}=0.3 \Omega . R_{a}$ is obtained by paralleling 4 resistances with $1.2 \Omega$. An RLC meter is used to measure the value of each component. Parasitic resistance of inductance is measured by ohmmeter and is seen as part of additional resistance. The equivalent series resistance (ESR) of the capacitor is also looked at as part of the additional resistance. The real model of capacitor and inductor are complicated and their nonlinearity and frequency dependency are not addressed here.

The experimental results are shown in Fig. 5. Figure 5(a) shows that the SEMD gives superior sound absorption over the open-circuit loudspeaker, and close inspection of impedance curves in Figs. 5(b) and 5(c) reveals that both reactance suppression and resistance adjustment contribute to the improvement. Circuit A (thin solid curves) has the largest capacitor and circuit $\mathrm{C}$ has the least. The result of circuit A shows the greatest reactance reduction when $f<f_{c A}=$ $195 \mathrm{~Hz}$ and the greatest extension of moderate absorption performance toward the DC frequency. However, circuits B and $\mathrm{C}$ lead to slightly higher absorption coefficients than that of circuit $\mathrm{A}$ in the effective frequency band, which is a reasonable trade-off. This trade-off originates from the fact that the electrically induced damping and reactance suppression are related quantities.

It is noted that, if the theoretical current or voltage output of the ideal operational amplifier (op-amp) exceeds the range available from the selected op-amp, the op-amp goes into saturation. In the current design, $R_{a}$ should be kept small and $\Delta R$ should avoid being too small and causing excessive acoustic damping (Zhang, 2012).

The overall resistance, capacitance, and inductance of the electrical circuit are maintained to be positive. Comparison of Tables II and III reveals that the most effective component in the shunt circuit is the capacitor. An NIC reduces the effective coil resistance from $6.3 \Omega$ to $1.6 \Omega$. There is little need to optimize the coil inductance using the NIC. Therefore, the primary function of the NIC is the suppression of the electrical resistance in the loudspeaker specimen, which is not designed for noise absorption. It is speculated that a tailor-made moving-coil device can make the NIC redundant and the SEMD becomes entirely passive.

\section{FURTHER EXTENSION OF ABSORPTIVE BAND BY HYBRID ABSORBER}

While the SEMD is effective on low-frequency noise absorption, there is always a need to absorb noise over a wide frequency band. In other words, an ideal absorber should be able to handle medium to high frequencies as well. The spectrum shown in Fig. 5(a) is good for the band from 150 to about $1200 \mathrm{~Hz}$ but the absorption coefficient drops above $650 \mathrm{~Hz}$. It is well known that traditional sound absorption material (SAM) made of glass fiber or rock wool absorb medium- and high-frequency noise well. The question then is whether the low-frequency absorber proposed here, SEMD, can work in tandem with SAM to achieve full-band sound absorption. Cobo et al. (2003) and

TABLE II. Technical data of loudspeaker model BG-17 according to http://www.visaton.com/en/ela/breitband/bg17_8.html.

\begin{tabular}{lcccc}
\hline \hline Dynamic moving mass & Nominal impedance & D.C. resistance, $R_{c}$ & Voice-coil inductance, $L_{c}$ & Mechanical Q \\
\hline $6.5 \mathrm{~g}$ & $8 \Omega$ & $6.3 \Omega$ & $700 \mu \mathrm{H}$ & 2.94 \\
Force factor $\mathrm{Bl}$ & Effective piston area & Resonance frequency & Diaphragm volume & Calculated $\delta$ \\
\hline $5.7 \mathrm{Tm}$ & $143 \mathrm{~cm}^{2}$ & $120 \mathrm{~Hz}$ & $2.7 \times 10^{5} \mathrm{~mm}^{3}$ & $1.67 \mathrm{Ns} / \mathrm{m}$ \\
\hline \hline
\end{tabular}



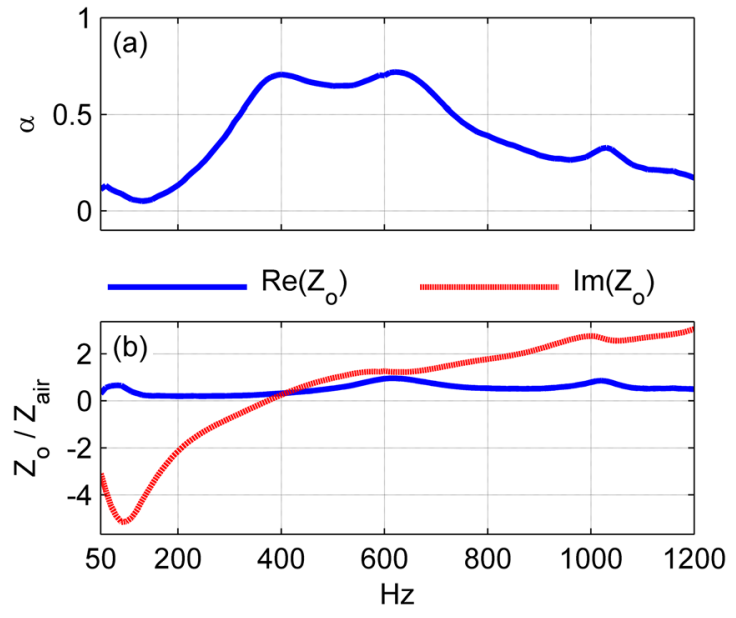

FIG. 4. (Color online) Sound absorption performance of open-circuit loudspeaker: (a) sound absorption coefficient, (b) normalized acoustic resistance (solid line), and reactance (dashed line).

Cobo et al. (2004) proposed a two-layer system by active loudspeaker and SAM with a sensing microphone to achieve broadband absorption, but this is unnecessary in our design.

Before finding suitable combinations, the sound absorption spectrum of the SEMD is compared with those of porous material and multi-layer micro-perforated panels with the same thickness. The schematics of the existing absorber designs are illustrated in Fig. 6. The comparison will be based on results sought from respective theories: the equivalent fluid model of Delany and Bazley (1970), modified by Miki (1990), is adopted to predict the absorption performance of porous material, and the properties of the MPP are calculated based
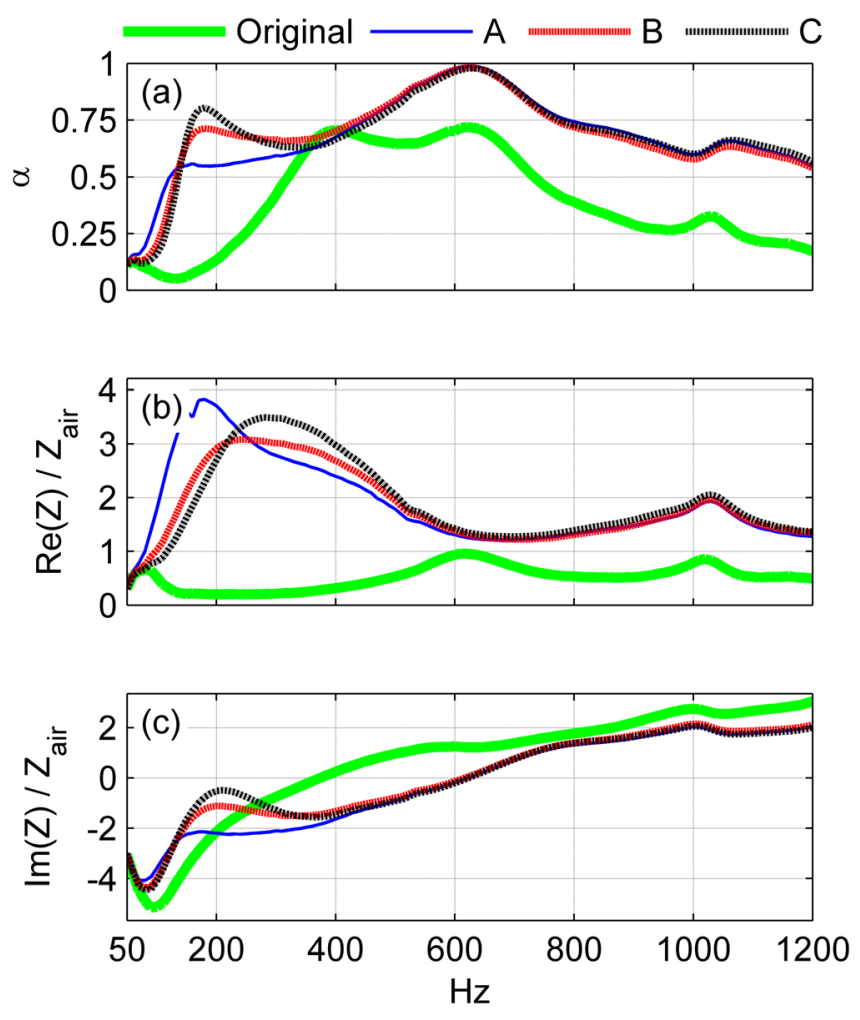

FIG. 5. (Color online) (a) Absorption coefficients, (b) mechanical resistance, and (c) normalized mechanical reactance of original loudspeaker and shunted counterparts. Legends apply to all plots.
TABLE III. Three sets of shunt circuit parameters used in testing.

\begin{tabular}{lccc}
\hline \hline & Set A & Set B & Set C \\
\hline$C(\mu \mathrm{F})$ & 1213 & 779 & 556 \\
$\Delta R(\Omega)$ & 1.6 & 1.6 & 1.6 \\
$\Delta L(\mu \mathrm{H})$ & 572 & 572 & 572 \\
$f_{c}(\mathrm{~Hz})$ & 190 & 238 & 282 \\
\hline \hline
\end{tabular}

on Maa (1998). Design optimization is carried out on each material numerically to maximize the absorption coefficient averaged over the frequency range from 50 to $1200 \mathrm{~Hz}$ with equal logarithmic intervals. This cost function puts higher weighting on the low-frequency region. Mathematically, the cost function is described as follows:

$$
\alpha_{\text {rand }}=\int_{50 \mathrm{~Hz}}^{1200 \mathrm{~Hz}} \alpha(f) \frac{d f}{f} .
$$

For the absorber with porous material, the flow resistivity is the only parameter to be optimized. The design variables of the double-layer perforated panels include cavity depths $D_{1}, D_{2}$, subject to the constraint of $D_{1}+D_{2}=60 \mathrm{~mm}$, the aperture diameters $d_{1}$ and $d_{2}$ (in the range of $0.2-2 \mathrm{~mm}$ ), the perforation ratios $\sigma_{1}$ and $\sigma_{2}$, while the panel thickness $t$ is chosen to be $0.5 \mathrm{~mm}$. The predicted results are shown in Fig. 7 and compared with the experimental results of the shunted loudspeaker. The latter uses parameter set B in Table III. All parameters are listed in the figure caption.

Figure 7 shows that the sound absorption performance of SEMD is satisfactory in a three-octave range between 140 and $1200 \mathrm{~Hz}$. Its absorption performance is better than SAM below $740 \mathrm{~Hz}$ and surpasses dMPP at low-frequency region. Experimental results of similar work (Boulandet and Lissek, 2010) are also shown; while Boulandet and Lissek produced better sound absorption at very low frequencies, the current device has superior broadband noise absorption performance. The comparison implies a good potential to utilize SEMD as a broadband noise absorber.

As sound absorption of SEMD is not as good as traditional absorbers at high frequencies, it is proposed to place an SEMD next to a single layer MPP. The resultant hybrid absorber is illustrated in Fig. 8. The specific acoustic admittance of the composite absorber is
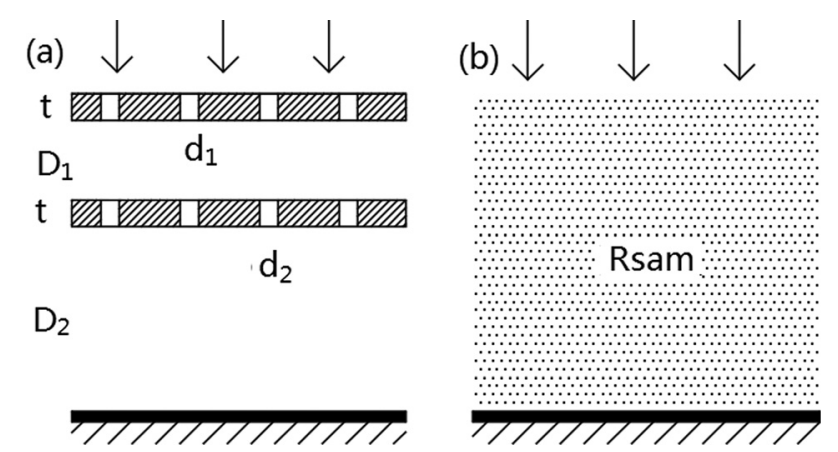

FIG. 6. Absorber designs used to compare with SEMD: (a) double-layer microperforated panel (dMPP) and (b) a single layer of porous sound absorption material (SAM). 


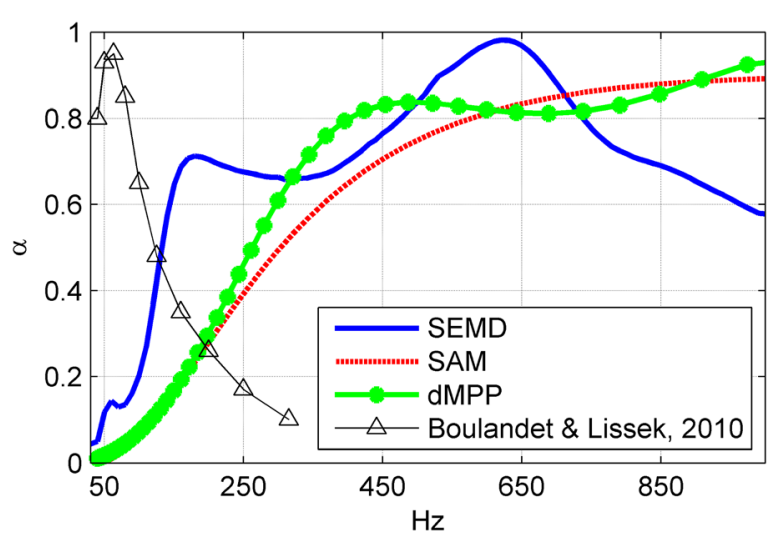

FIG. 7. (Color online) Sound absorption coefficients for SEMD (solid line), porous material (SAM, dashed line) and double-layer MPP (dMPP, dot-solid line). Flow resistivity of SAM $R_{\text {sam }}=28794 \mathrm{Ns} / \mathrm{m}^{4}$. Parameters of dMPP are $t=0.5 \mathrm{~mm}, d_{1}=2 \mathrm{~mm}, \sigma_{1}=1.465 \%, D_{1}=22.44 \mathrm{~mm}$, $d_{2}=0.246 \mathrm{~mm}, \sigma_{2}=0.365 \%, D_{2}=37.56 \mathrm{~mm}$. Data of triangle-solid line is from (Run \#8, Fig. 5, Boulandet and Lissek, 2010).

$$
\alpha_{\mathrm{SEMD} / / \mathrm{MPP}}=\left(\frac{A_{1}}{Z_{\mathrm{MPP}}}+\frac{A_{2}}{Z_{\mathrm{SEMD}}}\right) \frac{1}{A_{1}+A_{2}},
$$

where $Z_{\mathrm{MPP}}$ is the acoustic impedance of the perforated panel with the cavity, $Z_{\text {SEMD }}$ is the measured impedance of SEMD installed in the cavity of the same equivalent depth, while $A_{1}$ and $A_{2}$ are the cross-section areas occupied by the MPP and SEMD, respectively. The mechanism of hybrid absorber with MPP is discussed in detail in Wang and Huang (2011).

The predicted sound absorption performance is shown in Fig. 9. It is shown that the sound absorption coefficient is higher than $60 \%$ up to $1200 \mathrm{~Hz}$ in the hybrid design (solid line) instead of $650 \mathrm{~Hz}$ with an SEMD alone. The trade-off at lower frequencies is minimal. In other words, effective sound absorption is obtained across octaves from 150 to $1200 \mathrm{~Hz}$. It should be noted that the SEMD has not been optimized for this particular hybrid design and further improvement is expected.

The utility of SEMD-MPP absorber is further numerically demonstrated by its absorption of acoustics impulse. The impulse is constructed in time domain by a normalized $\operatorname{sinc}(a t)$ function whose is the Fourier transform of $\operatorname{rect}(f / a)$. By controlling $a$, the spectrum of impact noise is a rectangular across DC to $1200 \mathrm{~Hz}$. The waveform is used as normal incident wave. Although acoustic impulses may not appear exactly in the pattern of $\operatorname{sinc}(a t)$ (Coles et al., 1968; Akay, 1978) in practice, sinc function can be seen as a

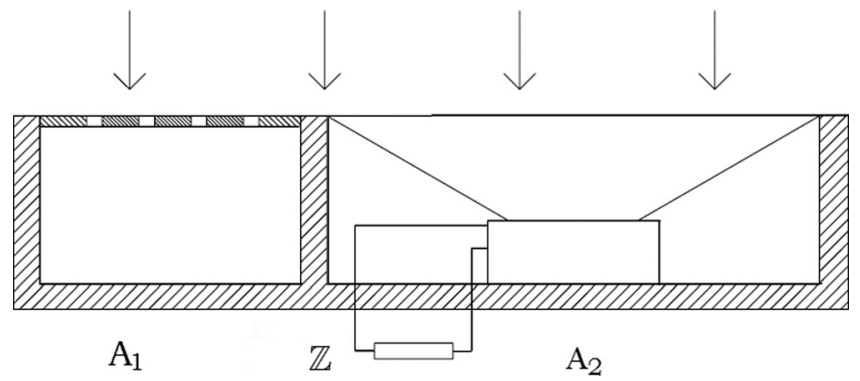

FIG. 8. Hybrid absorber by putting an SEMD next to a micro-perforated panel (MPP).

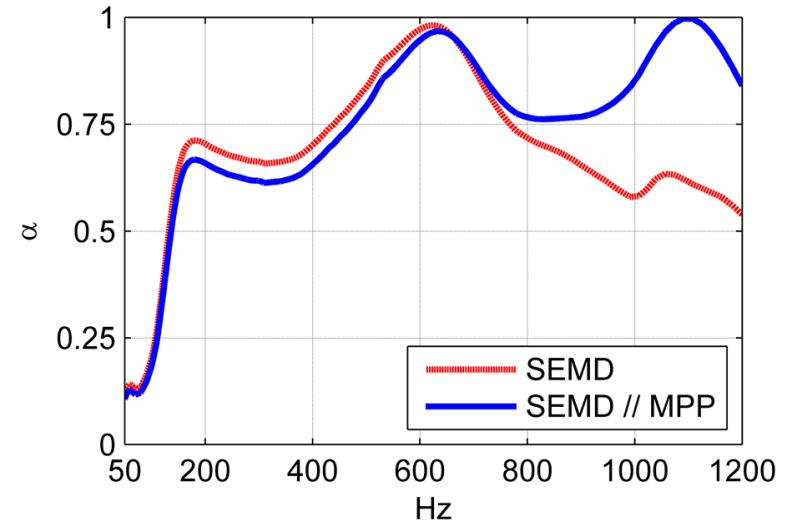

FIG. 9. (Color online) Absorption coefficient by SEMD and a hybrid SEMD-MPP absorber. MPP parameters are $t=1 \mathrm{~mm}, d=0.4 \mathrm{~mm}, \sigma=10 \%$, and it occupies $1 / 9$ of the total area.

symmetric B-type impulse noise (Coles et al., 1968; Akay, 1978; Henderson and Hamernik, 1986) and provides uniform energy distribution across the frequency band of interest. When $a$ approaches infinite, the impulse wave is approaching a Dirac delta function.

The peak pressure level of impulse noise is one of key parameters used to evaluate its hazard to human hearing (Coles et al., 1968; Henderson and Hamernik, 1986; Akay, 1978). It is therefore more important to reduce the peak magnitude than other spectral characters. The reduction of the impulse by an absorber may be evaluated by inspecting its reflection wave. Figure 10 shows that the peak amplitude of the band-limit impulse noise is reduced to $40 \%$ which means an $8 \mathrm{~dB}$ reduction in peak pressure level. It implies that the proposed absorber possesses the capability of protecting human hearing by reducing peak amplitude of impulsive noise thanks to its broadband absorption capability.

\section{CONCLUSIONS}

In this study, a shunt-circuit-based strategy to reduce acoustic reactance thus to achieve a broadband, lowfrequency sound absorber called the SEMD is proposed, and the predicted performance is validated experimentally using an impedance tube. The proposed device is compact in construction and it can reduce the magnitude of the system

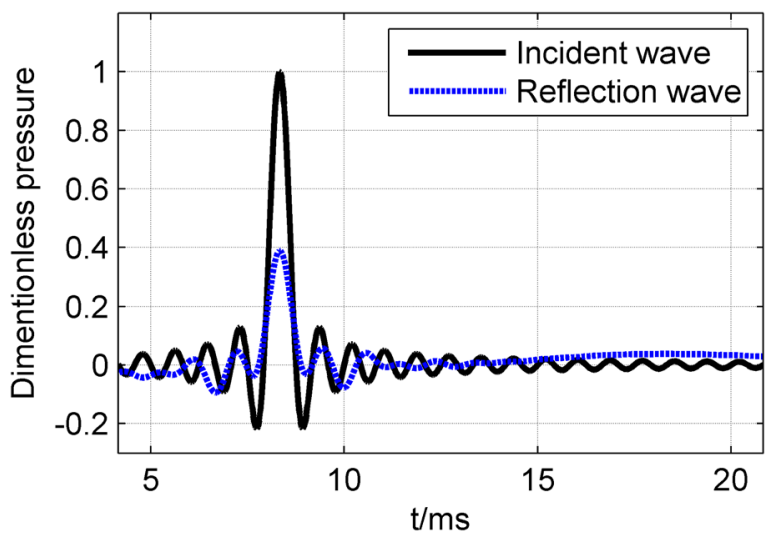

FIG. 10. (Color online) Absorption of acoustic impulse by SEMD-MPP absorber. 
reactance effectively. A shunt capacitor in series and reduced electrical resistance from the moving coil are found to be the key facilitators leading to such sound absorption improvements. Also, the shunt circuit introduces a virtual mechanical mass in the low-frequency region which counters the effect of high cavity stiffness in a compact sound absorber. The performance of the proposed device possesses a unique advantage in the low-frequency range when compared with porous sound absorption material and microperforated panels. Prediction on a hybrid SEMD-MPP sound absorber shows that the superior low-frequency performance can be combined with the good high-frequency performance of an MPP to achieve good sound absorption across 3 octaves. Such design is also able to attenuate reflection impulsive noise by $8 \mathrm{~dB}$.

\section{ACKNOWLEDGMENT}

The project is supported by a National Key Basic Research Scheme of China (Grant No. 2012CB720202).

Akay, A. (1978). "A review of impact noise," J. Acoust. Soc. Am 64, 977-987.

Behrens, S., Fleming, A. J., and Moheimani, S. (2003). "A broadband controller for shunt piezoelectric damping of structural vibration," Smart Mater. Struct. 12, 18-28.

Boulandet, R., and Lissek, H. (2010). "Optimization of electroacoustic absorbers by means of designed experiments," Appl. Acoust. 71, 830-842.

Chang, D., Liu, B., and Li, X. (2010). "An electromechanical low frequency panel sound absorber," J. Acoust. Soc. Am. 128, 639-645.

Chen, W. K. (2003). The Circuits and Filters Handbook (CRC Press, Boca Raton, FL), pp. 396-397.

Cheung, S. M. (2010). "Electric control of acoustic impedance," M. Phil thesis, The University of Hong Kong.

Chung, J. Y., and Blaser, D. A. (1980a). "Transfer function method of measuring in-duct acoustic properties. I. Theory," J. Acoust. Soc. Am. 68, 907-913.

Chung, J. Y., and Blaser, D. A. (1980b). "Transfer function method of measuring in-duct acoustic properties. II. Experiment,” J. Acoust. Soc. Am. 68, 914-921.

Cobo, P., Fernández, A., and Doutres, O. (2003). "Low-frequency absorption using a two-layer system with active control of input impedance," J. Acoust. Soc. Am. 114, 3211-3216.

Cobo, P., Pfretzschner, J., Cuesta, M., and Anthony, D. K. (2004). "Hybrid passive-active absorption using microperforated panels," J. Acoust. Soc. Am. 116, 2118-2125.

Coles, R. R. A., Garinther, G. R., Hodge, D. C., and Rice, C. G. (1968). "Hazardous exposure to impulse noise," J. Acoust. Soc. Am. 43, 336-343.

Delany, M. E., and Bazley, E. N. (1970). "Acoustical properties of fibrous absorbent materials," Appl. Acoust. 3, 105-116.

Eargle, J. (2003). Loudspeaker Handbook, 2nd ed. (Kluwer Academic, Boston, MA), Chaps. 1-4, pp. 1-63.

Fleming, A. J., Niederberger, D., Moheimani, S. O. R., and Morari, M. (2007). "Control of resonant acoustic sound fields by electrical shunting of a loudspeaker,” IEEE Trans. Control Syst. Technol. 15, 689-703.
Forward, R. L. (1979). "Electronic damping of vibrations in optical structures," Appl. Opt. 18, 690-697.

Fukada, E., Date, M., Kodama, H., and Oikawa, Y. (2005). "Elasticity control of curved piezoelectric polymer films," Ferroelectrics 320, 3-13.

Hagood, N. W., and von Flotow, A. (1991). "Damping of structural vibrations with piezoelectric materials and passive electrical networks," J. Sound. Vib. 146, 243-268.

Hansen, C. N. (2002). Understanding Active Noise Cancellation (Spon Press, London), Chaps. 2-4, pp. 9-81.

Henderson, D., and Hamernik, R. P. (1986). "Impulse noise: Critical review," J. Acoust. Soc. Am. 80, 569-584.

Huang, L. (2000). "A theory of reactive control of low frequency duct noise," J. Sound Vib. 238, 575-594.

Hunt, F. V. (1982). Electroacoustics: The Analysis of Transduction, and Its Historical Background (Acoustical Society of America, New York), Chap. 2, 92-102.

Ingard, K. U. (2008). Notes on Acoustics (Infinity Science Press, Hingham, MA), Chap. 4, pp. 105-147.

Kim, J., and Jung, Y. C. (2006). "Broadband noise reduction of piezoelectric smart panel featuring negative-capacitive-converter shunt circuit," J. Acoust. Soc. Am. 120, 2017-2025.

Kim, J., and Lee, J. K. (2002). "Broadband transmission noise reduction of smart panels featuring piezoelectric shunt circuits and sound-absorbing material," J. Acoust. Soc. Am. 112, 990-998.

Kodama, H., Date, M., Yamamoto, K., and Fukada, E. (2008). "A study of sound shielding control of curved piezoelectric sheets connected to negative capacitance circuits," J. Sound Vib. 311, 898-911.

Lissek, H., Boulandet, R., and René, P. J. (2009). "Shunt loudspeakers for modal control in rooms," in Proc. 16th International Congress on Sound and Vibration, July 5-9, Krakow, Poland.

Lissek, H., Boulandet, R., and Romain, F. (2011). "Electroacoustic absorbers: Bridging the gap between shunt loudspeakers and active sound absorption,” J. Acoust. Soc. Am. 129, 2968-2978.

Lissek, H., and Meynial, X. (2003). "A preliminary study of an isodynamic transducer for use in active acoustic materials," Appl. Acoust. 64, 917-930.

Maa, D. Y. (1998). "Potential of microperforated panel absorber," J. Acoust. Soc. Am. 104, 2861-2866.

Mechel, F. P. (2001). "Panel absorber," J. Sound Vib. 248, 43-70.

Miki, Y. (1990). "Acoustical properties of porous materials-Modifications of Delany-Bazley models,” J. Acoust. Soc. Jpn. (E) 11, 19-24.

Nelson, P. A., and Elliott, S. J. (1992). Active Control of Sound (Academic, London), p. 452.

Pietrzko, S., and Mao, Q. (2009). "Noise reduction in a duct using passive/ semiactive shunt loudspeakers," in 16th International Congress on Sound and Vibration, July 5-9, Krakow, Poland.

Swigert, C. J., and Forward, R. L. (1981). "Electronic damping of orthogonal bending modes in a cylindrical mast-theory," J. Spacecr. Rockets $\mathbf{1 8}$, 5-10.

Tajitsu, Y., Ueda, H., Tahara, K., Imoto, K., Date, M., and Fukada, E. (2007). "Basic studies of elasticity control of lead zirconate titanate (PZT) ceramic and its application to noise interception technology," Ferroelectrics 351, 43-50.

Tang, J., and Wang, K. W. (2001). "Active-passive hybrid piezoelectric networks for vibration control: Comparisons and improvement," Smart Mater. Struct. 10, 794-806.

Wang, C., and Huang, L. (2011). "On the acoustic properties of parallel arrangement of multiple micro-perforated panel absorbers with different cavity depths," J. Acoust. Soc. Am. 130, 208-218.

Zhang, Y. (2012). "Dynamic mass modification by electric circuits," M. Phil Thesis, The University of Hong Kong. 